\title{
Transceiver Design and Power Allocation for Full-Duplex MIMO Communication Systems with Spectrum Sharing Radar
}

\author{
Keshav Singh, Member, IEEE, Sudip Biswas, Member, IEEE, \\ Tharmalingam Ratnarajah, Senior Member, IEEE, and Faheem Khan, Member, IEEE,
}

\begin{abstract}
The networking paradigm of spectrum sharing is a promising technology to solve the spectrum paucity that has resulted from the exponential increase in the number of wireless devices and ubiquitous services. In light of the novel concept of Authorized/Licensed Shared Access, in this work, we consider the spectrum sharing between a collocated multiple-input-multipleoutput (MIMO) radar and a full-duplex (FD) MIMO cellular communication system consisting of a FD base station (BS) serving multiple downlink and uplink users simultaneously, without hindering the detection probability of the radar. The main objective is to develop an optimization technique at the cellular system for jointly designing the transceiver for the cellular BS and power allocation vectors for uplink users that can maximize the detection probability of radar, while guaranteeing a pre-defined quality-of-service for each user and power budget for the uplink users and BS. The original problem is non-convex and thus, we convert the non-convex problem into a secondorder cone and propose an iterative algorithm to find the optimal solution. Numerical results are then provided to demonstrate the feasibility of the spectral coexistence and show a scalable tradeoff in performance of both systems.
\end{abstract}

Index Terms-Full-duplex, multi-user, MIMO, radar, spectrum access, transceiver, optimization.

\section{INTRODUCTION}

Recent studies in areas of wireless communications reveal that the explosive growth in mobile data traffic due to the demand for ubiquitous, secure, and high data rate multimedia applications/services running on high-end client devices such as smart phones, tablets [1], etc., has led to a tremendous solicitation of limited radio resources such as spectrum. The key reason for highly inefficient spectrum utilization in traditional wireless communication systems is the static spectrum allocation [2]-[8], which leads to the problem of spectrum paucity. Further, by 2020, the number of connected devices is expected to jump to more than 20 billion. Hence, as a part of the global effort to address the overwhelming demand for wireless broadband capacity, government agencies around the world are promoting the use of shared spectrum between radar applications and wireless communications, to fully deliver on the promise of future $5 \mathrm{G}$ wireless communications systems.

Among the various spectrum access technologies, the main ones being considered are Cognitive Radios (CRs) [4], Licensed Shared Access/Authorized Shared Access (LSA/ASA)

This work was supported by the U.K. Engineering and Physical Sciences Research Council (EPSRC) under Grant EP/N014073/1 and the UK-India Education and Research Initiative Thematic Partnerships under grant number DST-UKIERI-2016-17-0060.

Keshav Singh, Sudip Biswas, and Tharmalingam Ratnarajah are with Institute for Digital Communications, School of Engineering, University of Edinburgh, Edinburgh, UK. (E-mail: \{k.singh, sudip.biswas, t.ratnarajah\}@ed.ac.uk)

Faheem Khan is with School of Computing and Engineering, University of Huddersfield, Queensgate, Huddersfield, UK. (E-mail: f.khan@hud.ac.uk)

The corresponding author of this paper is Sudip Biswas.
[9], [10] and Spectrum Access Systems (SAS). While LSA/ASA allows incumbents (primary licensed users) to offer their spectrum to licensees (secondary users (SU)), the SAS system is a slight modification of the existing LSA/ASA. Among many motivating factors for spectrum sharing, the President's National Broadband plan, which called to free up to $500 \mathrm{MHz}$ of federal-held spectrum by 2020 [11] is one of them. Some others motivating factors for LSA/ASAbased spectrum sharing are the report on efficient spectrum utilization by President's Council of Advisers on Science and Technology (PCAST), which focused to share $1.0 \mathrm{GHz}$ of government-held spectrum [12], the low utilization of huge amounts of spectrum held by the federal incumbents, for example: the $3.55-3.65 \mathrm{GHz}, 5.25-5.35 \mathrm{GHz}$, and $5.47-5.725$ $\mathrm{GHz}$ bands [13] and the National Telecommunication and Information Administration's (NTIA's) 2010 Fast Track Report [14], which stated that the $3550-3650 \mathrm{MHz}$ band, currently used for military and satellite operations, is a possible candidate for spectrum sharing between military radars and broadband wireless access communication systems. Further, the Third Generation Partnership Program (3GPP) Release 12 standard is also currently considering to make use of $3.5 \mathrm{GHz}$ band for small cell deployment and network densification [15]. In this regard, while as an experimental validation, Reed et al. showed the operation of Time-Division Long-Term Evolution (TD-LTE) in the presence of pulsed interfering signals in the $3.55-3.65 \mathrm{GHz}$ frequency band in [16], in [17] an overview of some of the techniques that can be used for enabling efficient co-existence of commercial LTE and radar systems in the $3.5 \mathrm{GHz}$ band were presented. Similarly, in Europe, LSA has been identified by the European Commission (EC) and Conférence Européenne des Postes et des Télécommunications as the common basis for voluntary sharing within existing licenses in general, and especially for the implementation of Mobile/Fixed Communication Networks in military bands.

Apart from spectrum sharing, efficient spectrum utilization is another key issue that needs to be addressed. The spectrum efficiency (SE) in wireless communication systems can be significantly improved by operating in a full-duplex (FD) mode, which is another technology that is being considered for $5 \mathrm{G}$ and beyond. A FD transceiver can receive and transmit at the same time and the same frequency. However, the selfinterference caused by the signal leakage dominates the performance of FD system. Thanks to the advancement in interference cancellation techniques and transmit/receive antenna isolation such as antenna design, and analog and digital domain self-interference cancellation techniques [18]-[20], that have enabled FD transceivers to sufficiently combat the interference. However, due to the inherent imperfection of the transmit and receive chains [21]-[24], a residual self-interference is 
still required to be taken into account. Furthermore, the cochannel interference (CCI), i.e., the interference from the uplink (UL) to downlink (DL) users, is another challenge in cellular communications for the gainful use of FD technology. Therefore, in the light of the above discussions, the design of beamforming techniques while considering the impact of the CCI, as well as the self-interference jointly [25], [26] will be an effective remedy for mitigating the interferences.

1) Related Work: Recently, adequate studies have focused on the subject of spectrum sharing between radars and communication systems [27]-[40]. In [27], the possibility of spectrum sharing with rotating radar was addressed, the problem of spectrum sharing between a primary pulsed, search radar and a secondary 802.11 WLAN was investigated in [28]. Similarly, the authors in [30], [31] studied cooperative sensing based opportunistic spectrum access (OSA) between a rotating radar and a cellular system, where the SU in communication system is permitted to transmit signals if and only if (iff) the space and frequency spectra are not utilized by the radar. However, the radar and communication systems cannot work simultaneously with the methods investigated in [30], [31]. With regards to the joint operation of radar and cellular systems, the null-space projection (NSP) method was used in [35], [36] to design the precoder in order to project the radar signal to nullify the interference for the communication system. However, the interference received by the radar from the communication system was ignored for simplicity. While ignoring the interference towards radar simplifies the analysis, in practice the interference temperature is an integral criteria in the design of underlay networks. Especially in the SAS, where there is a restriction on interference from the lower tiers to the upper ones, the upper tiers can set predefined tolerable interference temperature limits.

Furthermore, if the cellular system operates in FD mode, the level of interference towards the radar will be even more and will play a significant role in determining the detection probability of the co-existed radar. Recently, FD cellular systems have been extensively considered in beamformer design problems in [41]-[43]. However, most works on FD consider only single-users. Further, no work is yet to consider a FD multi-user MIMO cellular system that shares the spectrum of a MIMO radar.

2) Key Contributions: While spectrum sharing is a lucrative prospect from the cellular network's perspective, target detection comes at the forefront for radar applications. Considering the seriousness of radar applications, the problem of spectrum sharing with commercial systems becomes critical owing to security concerns. In this paper we will restrict our discussion to spectrum sharing between cellular systems and radar. The design goals for the sharing mechanism are as follows: 1) by sharing the spectrum with cellular systems, radar should be able to decide the amount of interference it can tolerate so that its detection performance is above a certain predefined threshold, and 2) the solution mechanisms should not be based on any specific regional regulatory requirement.

Based on the aforementioned discussion and consideration around the globe to share the radar spectrum with commercial cellular networks, in this paper, the main focus is to design

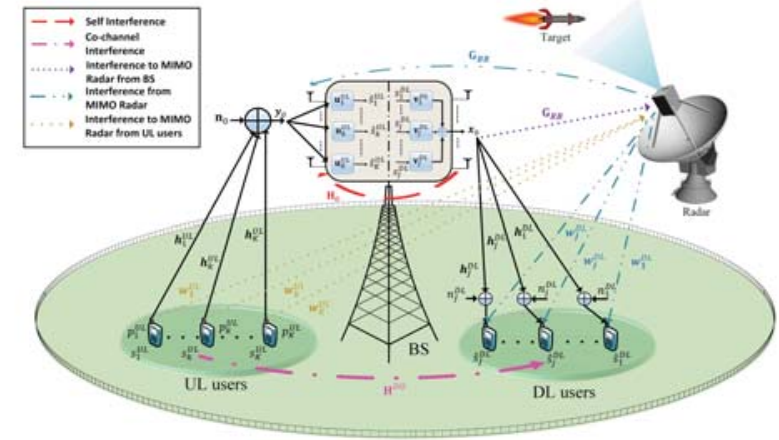

Fig. 1: FD MIMO communication system with spectrum sharing MIMO radar.

transceivers for a FD cellular system for effective spectrum sharing between a collocated MIMO radar and a FD multiuser MIMO cellular system. The cellular system employs a FD MIMO base station (BS), which transmits and receives signals at the same time and the same frequency, to and from single antenna equipped $J$ DL and $K$ UL users, which operate in half-duplex (HD) mode, simultaneously. To mitigate the interference of the cellular system towards the radar, we design transceivers at the cellular system which takes into consideration a tolerable interference temperature limit set by the radar. The transceiver design technique also takes into account the interference from the radar towards the cellular system. This allows the cellular system to transmit within the radar's spectrum resources and meet the QoS of its users.

By applying the generalized likelihood ratio test [44], [45], we formulate the optimization problem of maximizing the detection probability of a MIMO radar subject to minimum quality-of-service (QoS), i.e., SINR requirements for each DL and UL users and transmit power budget for the BS and UL users. In order to gain further insights into the structure of the iterative algorithm, we analyze the optimal beamforming and power allocation schemes theoretically under two different regimes: 1) an interference-dominated regime and 2) a BS noise-dominated regime. Our analyses show that the optimal beamforming strategies behave similar to the water-filling approach. Finally, computer simulations are performed to demonstrate the feasibility of the spectral coexistence, which illustrate a trade-off in the performance of both systems.

The remainder of this paper is structured as follows. The network architecture is presented in Section II. The problem formulation for maximizing the detection probability of the MIMO radar through joint transceiver design is illustrated in Section III, followed by the problem transformation. An iterative algorithm is then presented in Section IV. In addition, we analyze the optimal beamforming and power allocation strategies under different operating regimes in Section V. While Section VI describes the computational complexity of the algorithms, numerical results and conclusions are provided in Section VII and Section VIII, respectively.

3) Notations: Boldface capital and small letters denote matrices and vectors, respectively. Transpose and conjugate transpose are respectively denoted by $(\cdot)^{T}$ and $(\cdot)^{H} \cdot\|\mathbf{A}\|_{F}$ and $\|\mathbf{a}\|_{2}$ denote the Frobenius norm of a matrix $\mathbf{A}$ and the Euclidean norm of a vector a, respectively. The Kronecker 
product is $\otimes$, while $\perp$ denotes statistical independence. The matrices $\mathbf{I}_{N}$ and $\mathbf{O}_{M \times N}$ denote an $N \times N$ identity matrix and an $M \times N$ zero matrix, respectively. The notations $E(\cdot)$ and $\operatorname{tr}(\cdot)$ refer to expectation and trace, respectively. Further, $\operatorname{diag}(\mathbf{A})$ generates a diagonal matrix with the same diagonal element as $\mathbf{A}$, whereas matrix $\mathbf{A}$ is said to be positive semidefinite (PSD) if $\mathbf{A} \succeq 0$.

\section{Network Architecture AND Preliminaries}

\section{A. FD Multi-User MIMO Cellular System}

We consider an FD MIMO communication system, which consists of an FD MIMO BS with $M_{0}$ transmit and $N_{0}$ receive antennas, $J$ DL users, and $K$ UL users. All DL and UL users operate in half-duplex mode and each DL and UL users are equipped with single antenna. The MIMO cellular system coexists with a MIMO radar as shown in Fig. 1.

We define $\mathbf{h}_{k}^{U L} \in \mathbb{C}^{N_{0} \times 1}$ and $\mathbf{h}_{j}^{D L} \in \mathbb{C}^{1 \times M_{0}}$ the channels from $k$-th UL user to BS and from BS to the $j$-th DL user, respectively. The self-interference channel at the FD BS and the CCI channel between the $k$-th UL and $j$-th DL users are denoted as $\mathbf{H}_{0} \in \mathbb{C}^{N_{0} \times M_{0}}$ and $h_{j k}^{D U}$, respectively. It is assumed that all links in the network are Rayleigh flatfading and statistically independent with each other. Besides, it is assumed that the BS has full knowledge of the channel state information (CSI) of all links. The time slot index is $l=1,2, \ldots, L$, where $L$ indicated the length of the communication frame. The signal received at the BS and the $j$-th DL user at $l$-th time slot can be written, respectively, as

$$
\begin{gathered}
\mathbf{y}_{0}(l)=\sum_{k=1}^{K} \sqrt{P_{k}^{U L}} \mathbf{h}_{k}^{U L} s_{k}^{U L}(l) \\
+\mathbf{H}_{0} \mathbf{x}_{0}(l)+\sqrt{P_{R}} \mathbf{G}_{B R} \mathbf{s}_{R}(l)+\mathbf{n}_{0}(l) ;(1) \\
y_{j}^{D L}(l)=\mathbf{h}_{j}^{D L} \mathbf{x}_{0}(l)+\sum_{k=1}^{K} \sqrt{P_{k}^{U L}} h_{j k}^{D U} s_{k}^{U L}(l) \\
+\sqrt{P_{R}} \mathbf{w}_{j}^{D L} \mathbf{s}_{R}(l)+n_{j}^{D L}(l)
\end{gathered}
$$

where $\mathbf{G}_{B R} \in \mathbb{C}^{N_{0} \times R_{T}}$ and $\mathbf{w}_{j}^{D L} \in \mathbb{C}^{1 \times R_{T}}$ denote the interference channels from radar transmitter to $\mathrm{BS}$ and $j$ th DL user, respectively. $\mathbf{x}_{0}$ is the signal transmitted from the FD BS, defined as $\mathbf{x}_{0}=\sum_{j=1}^{J} \mathbf{v}_{j}^{D L} s_{j}^{D L}(l)$, where $\mathbf{v}_{j}^{D L} \in \mathbb{C}^{M_{0} \times 1}$ denotes the precoding vector and $s_{j}^{D L}(l)$ stands for the communication symbol for the $j$-th DL user. The communication symbol for the $k$-th UL user is presented by $s_{k}^{U L}(l)$. It is assumed that these symbols are independent and identically distributed (i.i.d.) with unit power, i.e., $\mathbb{E}\left[s_{k}^{U L}(l)\left(s_{k}^{U L}(l)\right)^{H}\right]=1$ and $\mathbb{E}\left[s_{j}^{D L}(l)\left(s_{j}^{D L}(l)\right)^{H}\right]=1$. The terms $\mathbf{n}_{0} \in \mathbb{C}^{N_{0}}$ and $n_{j}^{D L}$ in (1) and (2) denote the additive white Gaussian noise (AWGN) with zero mean and covariance matrix $\mathbf{R}_{0}=\sigma_{0}^{2} \mathbf{I}_{N_{0}}$ and $\sigma_{j}^{2}$ at the BS and the $j$-th DL user, respectively. From (1) and (2), the received signalto-interference-plus-noise ratio (SINR) at the BS for $k$-th UL user and at the $j$-th DL user can be given as

$$
\begin{gathered}
\Upsilon_{k}^{U L}=\frac{P_{k}^{U L}\left\|\mathbf{h}_{k}^{U L}\right\|^{2}}{\left\{\begin{array}{c}
\left.\sum_{i=1, i \neq k}^{K} P_{i}^{U L}\left\|\mathbf{h}_{i}^{U L}\right\|^{2}+\sum_{j=1}^{J}\left\|\mathbf{H}_{0} \mathbf{v}_{j}^{D L}\right\|^{2}\right\} \\
+P_{R}\left\|\mathbf{G}_{B R}\right\|^{2}+N_{0} \sigma_{0}^{2}
\end{array}\right\}} \\
\Upsilon_{j}^{D L}=\frac{\left|\mathbf{h}_{j}^{D L} \mathbf{v}_{j}^{D L}\right|^{2}}{\left\{\begin{array}{c}
\left.\sum_{l=1, l \neq j}^{J}\left|\mathbf{h}_{l}^{D L} \mathbf{v}_{l}^{D L}\right|^{2}+\sum_{k=1}^{K} P_{k}^{U L}\left|h_{j k}^{D U}\right|^{2}\right\} \\
+P_{R}\left\|\mathbf{w}_{j}^{D L}\right\|^{2}+\sigma_{j}^{2}
\end{array}\right\}} .
\end{gathered}
$$

\section{B. MIMO Radar}

We consider a colocated MIMO radar $^{1}$ consisting of $R_{T}$ transmit and $R_{R}$ receive antennas. Without loss of generality, we consider the echo wave in a single range-Doppler bin of the colocated MIMO radar. The discrete time signal vector received by the MIMO radar at an angle $\theta$ from a point like target in the far field can be expressed as

$$
\begin{aligned}
\mathbf{y}_{R}=\alpha \sqrt{P_{R}} \mathbf{V}(\theta) \mathbf{s}_{R} & +\mathbf{G}_{R B} \sum_{j=1}^{J} \mathbf{v}_{j}^{D L} s_{j}^{D L} \\
& +\sum_{k=1}^{K} \sqrt{P_{k}^{U L}} \mathbf{w}_{k}^{U L} s_{k}^{U L}+\mathbf{n}_{R},
\end{aligned}
$$

where $\mathbf{G}_{R B} \in \mathbb{C}^{R_{R} \times M_{0}}$ and $\mathbf{w}_{k}^{U L} \in \mathbb{C}^{R_{R} \times 1}$ are the interference channels from BS to radar receiver and from $k$-th UL user to radar receiver, respectively. While $P_{R}$ and $P_{k}^{U L}$ are the transmit power of radar and the $k$-th UL user, respectively, $\mathbf{s}_{R}(l) \in \mathbb{C}^{R_{T} \times 1}$ denotes the MIMO radar waveform ${ }^{2}$ at $l$ th time slot and it is assumed to be orthogonal [32], [46], i.e., $\mathbb{E}\left[\mathbf{s}_{R}(l)\left(\mathbf{s}_{R}(l)\right)^{H}\right]=\frac{1}{L} \sum_{l=1}^{L}\left[\mathbf{s}_{R}(l)\left(\mathbf{s}_{R}(l)\right)^{H}\right]=\mathbf{I} . \alpha$ indicates the complex path loss of the radar-target-radar path including the propagation loss and the coefficient of reflection, $\mathbf{n}_{R} \sim \mathcal{C N}\left(0, \sigma_{R}^{2} \mathbf{I}_{R}\right)$ is the white Gaussian noise, and $\mathbf{V}(\theta)$ denotes the transmit-receive steering matrix expressed as

$$
\mathbf{V}(\theta) \triangleq \mathbf{v}_{R}(\theta) \mathbf{v}_{T}^{T}(\theta) .
$$

Here, $\mathbf{v}_{T} \in \mathbb{C}^{R_{T} \times 1}$ and $\mathbf{v}_{R} \in \mathbb{C}^{R_{R} \times 1}$ express transmit and receive steering vectors of radar antenna array. We adopt the model defined in [9] and define $\mathbf{V}_{i r}(\theta)$ with assumptions $R_{R}=R_{T}=R, \mathbf{v}_{R}(\theta)=\mathbf{v}_{T}(\theta)=\mathbf{v}(\theta)$, and $\mathbf{V}_{i r}(\theta)=$ $\mathbf{v}_{i}(\theta) \mathbf{v}_{r}(\theta)=\exp \left(-j \omega \tau_{i r}(\theta)\right)$ as follows:

$$
\mathbf{V}_{i r}(\theta)=\exp \left(-j \frac{2 \pi}{\lambda}[\sin (\theta) ; \cos (\theta)]^{T}\left(\mathbf{z}_{i}+\mathbf{z}_{r}\right)\right)
$$

Here, $\mathbf{V}_{i r}(\theta)$ denotes the $i$-th element at the $r$-th column of the matrix $\mathbf{V}$ and $\mathbf{z}_{i}=\left[z_{i}^{1} ; z_{i}^{2}\right]$ is the location of the $i$-the element of the antenna array. $\omega$ and $\lambda$ express the frequency and the wavelength of the carrier. For simplicity, we ignore the interference by clutter and false targets. For the sake of convenience, hereinafter we drop the time slot index, $l=1,2, \ldots, L$.

\section{Dection Probability of MIMO Radar}

The detection probability of MIMO radar is affected by the interference from the cellular system BS and UL users to the radar receiver. We use the generalized likelihood ratio test (GLRT) [44], [45], which has the advantage of replacing the unknown parameters with their maximum likelihood (ML) estimates, for determining the detection probability. The asymptotic detection probability of the MIMO radar under the Neyman-Pearson criterion can be defined as [35], [46]

$$
P_{D}=1-\mathfrak{F}_{\mathcal{X}_{2}^{2}(\rho)}\left(\mathfrak{F}_{\mathcal{X}_{2}^{2}}^{-1}\left(1-P_{F A}\right)\right),
$$

where $\mathfrak{F}_{\mathcal{X}_{2}^{2}(\rho)}$ denotes the noncentral chi-squared distribution function with two degrees of freedom (DoF) and noncentrality parameter $\rho . \mathfrak{F}_{\mathcal{X}_{2}^{2}}^{-1}$ represents the inverse function of chi-squared distribution with two DoFs. The noncentrality parameter $\rho$ for $\mathcal{X}_{2}^{2}(\rho)$ can be written as [47]

\footnotetext{
${ }^{1}$ The colocated MIMO radar is considered in this work as it gives better spatial resolution and target detection capabilities as compared to the widely spaced radar [32].

${ }^{2}$ For convenience, we assume that the MIMO radar uses the same symbol
} rate as the UL-DL communications system. 


$$
\begin{array}{rl}
\rho=|\alpha|^{2} & L P_{R} \operatorname{tr}\left(\mathbf { V } \mathbf { V } ^ { H } \left(\mathbf{G}_{R B} \sum_{j=1}^{J} \mathbf{v}_{j}^{D L}\left(\mathbf{v}_{j}^{D L}\right)^{H} \mathbf{G}_{R B}^{H}\right.\right. \\
& \left.\left.+\sum_{k=1}^{K} P_{k}^{U L} \mathbf{w}_{k}^{U L}\left(\mathbf{w}_{k}^{U L}\right)^{H}+\sigma_{R}^{2} \mathbf{I}_{R}\right)^{-1}\right) .
\end{array}
$$

\section{Framework For Spectrum Sharing}

To realize the goal of simultaneous co-existence between a radar and a cellular system, in this work we assume that cellular systems can transmit at power levels that do not exceed the allowed interference threshold set by the radar. This method, while providing the cellular system with connectivity, also allows the radar to meet its desired QoS. In the following, we formulate the transceiver design problem that will take into account both the interference from the radar to the cellular system and vice-versa. For the spectral co-existence, the radar shares $\mathcal{L}$ interference channels, denoted as $\mathbf{W}_{l} \in \mathbb{C}^{N_{B S+U E} \times R_{T}}$ with the cellular system, where $N_{B S+U E}=1+J$ and $l=1, \ldots, \mathcal{L}$. Accordingly, $\left\{\mathbf{W}_{B R}^{D L}, \mathbf{W}_{j}^{D L}\right\} \subseteq \mathbf{W}_{l}$, with $j=1, \ldots, J$.

\section{A. Problem Formulation for Maximizing Detection Probability}

This section presents a joint power allocation scheme for $\mathrm{UL}$ users and precoder design for the BS for maximizing the detection probability of MIMO radar. The UL users and the BS generate interference towards the MIMO radar. Accordingly, the interference from the UL users and the BS to the $r$-th antenna of MIMO radar is given as

$$
u_{r}=\mathbf{g}_{R B} \sum_{j=1}^{J} \mathbf{v}_{j}^{D L} \mathbf{s}_{j}^{D L}+\sum_{k=1}^{K} \sqrt{P_{k}^{U L}} \mathbf{w}_{k}^{U L} \mathbf{s}_{k}^{U L},
$$

where $\mathbf{g}_{R B} \in \mathbb{C}^{1 \times M_{0}}$. From (10), the interference-to-noiseratio (INR) at the $r$-th receive antenna of radar is given by

$$
I_{r}=\left|u_{r}\right|^{2} / \sigma_{R}^{2}
$$

Furthermore, the total transmit power of the BS and UL users are given as

$$
\begin{aligned}
T_{B S} & =\sum_{j=1}^{J}\left\|\mathbf{v}_{j}^{D L}\right\|^{2}, \\
T^{U L} & =\sum_{k=1}^{K} P_{k}^{U L} .
\end{aligned}
$$

Here, the main objective is to improve the detection performance of radar by jointly optimizing the precoder vector $\mathbf{v}_{j}^{D L}$ for DL users and power allocation for UL users, while maintaining desired QoS of each UL and DL users and power budget for UL users and the BS.

\section{B. Optimization Problem Formulation}

From (3), (4), and (11)-(13), the optimization problem for maximizing detection probability performance of MIMO radar is formulated as follows:

(P1)

$$
\max _{\mathbf{v}^{D L}, \mathbf{p}^{U L}} P_{D}
$$

subject to

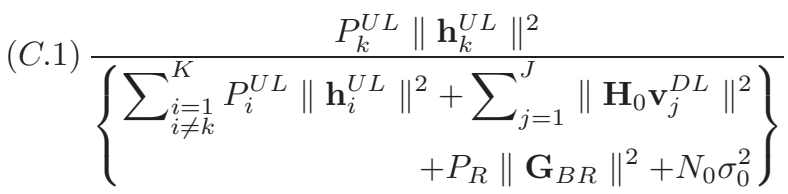

$$
\begin{aligned}
& \geq \Gamma_{k, \text { min }}^{U L}, \forall k \text {; }
\end{aligned}
$$

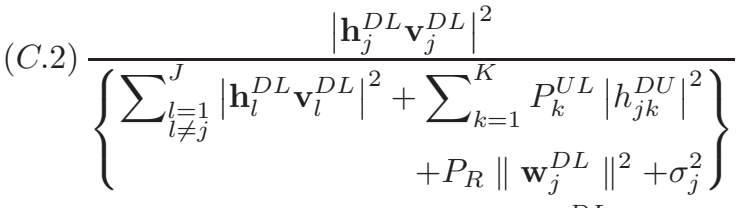
$\geq \Gamma_{j, \text { min }}^{D L}, \forall j$

(C.3) $\sum_{j=1}^{J}\left\|\mathbf{v}_{j}^{D L}\right\|^{2} \leq P_{B S, \max } ;$

(C.4) $\sum_{k=1}^{K} P_{k}^{U L} \leq P_{U L, \max }$

where $\Gamma_{k, \text { min }}^{U L}$ in (14b) and $\Gamma_{j, \min }^{D L}$ in $(14 \mathrm{c})$ are the minimum QoS requirement for the $k$-th UL and $j$-th DL users, respectively, while $P_{B S, \max }$ in (14d) and $P_{U L, \max }$ in $(14 \mathrm{e})$ denote the maximum available power budget for the BS and the UL users, respectively. Further, $\mathbf{v}^{D L}=\left\{\mathbf{v}_{j}^{D L}\right\}, \forall j$, and $\mathbf{p}^{U L}=\left\{P_{k}^{U L}\right\}, \forall k$, represent the set of all transmit beamforming vectors for DL users and power allocation for UL users, respectively.

Since the detection probability, $P_{D}$, is a monotonically increasing function with the non-centrality parameter, $\rho$ [47], we can equivalently rewrite the optimization problem (P1) as

$$
\text { (P2) } \begin{aligned}
\max _{\mathbf{v}^{D L}, \mathbf{p}^{U L}} & \operatorname{tr}\left(\mathbf { V } \mathbf { V } ^ { H } \left(\mathbf{G}_{R B} \sum_{j=1}^{J} \mathbf{v}_{j}^{D L}\left(\mathbf{v}_{j}^{D L}\right)^{H} \mathbf{G}_{R B}^{H}\right.\right. \\
+ & \left.\left.\sum_{k=1}^{K} P_{k}^{U L} \mathbf{w}_{k}^{U L}\left(\mathbf{w}_{k}^{U L}\right)^{H}+\sigma_{R}^{2} \mathbf{I}_{R}\right)^{-1}\right)
\end{aligned}
$$$$
\text { subject to } \quad(C .1)-(C .4) \text {. }
$$

The optimization problem (P2) is non-convex and intractable because the objective function is apparently non-concave. We provide the transformation of the objective function into a convex form as follows:

1) Transformation of objective function: The objective function in (P2) is non-convex, and thus it is required to transform this non-convex function into a convex one. By letting

$\boldsymbol{\Omega}=\mathbf{G}_{R B} \sum_{j=1}^{J} \mathbf{v}_{j}^{D L}\left(\mathbf{v}_{j}^{D L}\right)^{H} \mathbf{G}_{R B}^{H}+\sum_{k=1}^{K} P_{k}^{U L} \mathbf{w}_{k}^{U L}\left(\mathbf{w}_{k}^{U L}\right)^{H}+\sigma_{R}^{2} \mathbf{I}_{R}$,

the lower bound on the objective function in (P2) can be obtained. $\Omega$ and $\mathbf{V} \mathbf{V}^{H}$ are positive-definite, which reveals the following relation:

$$
\operatorname{tr}\left(\mathbf{V} \mathbf{V}^{H} \boldsymbol{\Omega}^{-1} \boldsymbol{\Omega}\right) \leq \operatorname{tr}\left(\mathbf{V} \mathbf{V}^{H} \boldsymbol{\Omega}^{-1}\right) \operatorname{tr}(\boldsymbol{\Omega})
$$

From (16), we have

$$
\begin{aligned}
\operatorname{tr}\left(\mathbf{V} \mathbf{V}^{H} \boldsymbol{\Omega}^{-1}\right) & \geq \frac{\operatorname{tr}\left(\mathbf{V} \mathbf{V}^{H}\right)}{\operatorname{tr}(\boldsymbol{\Omega})} ; \\
& =\frac{R^{2}}{\left\{\begin{array}{c}
\operatorname{tr}\left(\mathbf{G}_{R B} \sum_{j=1}^{J} \mathbf{v}_{j}^{D L}\left(\mathbf{v}_{j}^{D L}\right)^{H} \mathbf{G}_{R B}^{H}\right) \\
\quad+\sum_{k=1}^{K} P_{k}^{U L}\left\|\mathbf{w}_{k}^{U L}\right\|^{2}+R \sigma_{R}^{2}
\end{array}\right\}} .
\end{aligned}
$$

Now, the term $\operatorname{tr}\left(\mathbf{G}_{R B} \sum_{j=1}^{J} \mathbf{v}_{j}^{D L}\left(\mathbf{v}_{j}^{D L}\right)^{H} \mathbf{G}_{R B}^{H}\right)$ can be explicitly rewritten as 


$$
\begin{aligned}
\operatorname{tr}\left(\mathbf{G}_{R B} \sum_{j=1}^{J} \mathbf{v}_{j}^{D L}\left(\mathbf{v}_{j}^{D L}\right)^{H} \mathbf{G}_{R B}^{H}\right) & \\
& =\sum_{r=1}^{R}\left|\mathbf{g}_{R B, r} \sum_{j=1}^{J} \mathbf{v}_{j}^{D L} s_{j}^{D L}\right|^{2},
\end{aligned}
$$

where $\mathbf{g}_{R B, r} \in \mathbb{C}^{1 \times M_{0}}$ is the $r$-th row vector of matrix $\mathbf{G}_{R B}$. From (17) and (18), the objective function in (15) can be equivalently written as

$$
\begin{aligned}
\max _{\mathbf{v}^{D L}, \mathbf{p}^{U L}} \operatorname{tr}\left(\mathbf{V} \mathbf{V}^{H}\right. & \left(\mathbf{G}_{R B} \sum_{j=1}^{J} \mathbf{v}_{j}^{D L}\left(\mathbf{v}_{j}^{D L}\right)^{H} \mathbf{G}_{R B}^{H}\right. \\
& \left.\left.+\sum_{k=1}^{K} P_{k}^{U L} \mathbf{w}_{k}^{U L}\left(\mathbf{w}_{k}^{U L}\right)^{H}+\sigma_{R}^{2} \mathbf{I}_{R}\right)^{-1}\right) \\
\approx & \min _{\mathbf{v}^{D L}, \mathbf{p}^{U L}} \sum_{r=1}^{R}\left|\mathbf{g}_{R B, r} \sum_{j=1}^{J} \mathbf{v}_{j}^{D L} s_{j}^{D L}\right|^{2} \\
& +\sum_{k=1}^{K} P_{k}^{U L}\left\|\mathbf{w}_{k}^{U L}\right\|^{2},
\end{aligned}
$$

It can be observed from (19) that the original objective function of maximizing the detection probability of the radar is equivalent to minimizing the total interference from UL users and BS to the MIMO radar. Now, using (19) and epigraph method [48], we can reformulate the problem (P2) as follows:

(P3)

$$
\min _{\mathbf{v}^{D L}, \mathbf{p}^{U L}, \Psi_{U L}, \Psi_{D L}} \Psi_{U L}+\Psi_{D L}
$$

subject to

$$
\begin{aligned}
& \text { (C.1) } \sum_{i=1, i \neq k}^{K} P_{i}^{U L}\left\|\mathbf{h}_{i}^{U L}\right\|^{2}+\sum_{j=1}^{J}\left\|\mathbf{H}_{0} \mathbf{v}_{j}^{D L}\right\|^{2} \\
& +P_{R}\left\|\mathbf{G}_{B R}\right\|^{2}+N_{0} \sigma_{0}^{2} \leq \frac{1}{\Gamma_{k, \text { min }}^{U L}} P_{k}^{U L}\left\|\mathbf{h}_{k}^{U L}\right\|^{2}, \quad \forall k \\
& \text { (C.2) } \sum_{l=1, l \neq j}^{J}\left|\mathbf{h}_{l}^{D L} \mathbf{v}_{l}^{D L}\right|^{2}+\sum_{k=1}^{K} P_{k}^{U L}\left|h_{j k}^{D U}\right|^{2} \\
& +P_{R}\left\|\mathbf{w}_{j}^{D L}\right\|^{2}+\sigma_{j}^{2} \leq \frac{1}{\Gamma_{j, \min }^{D L}}\left|\mathbf{h}_{j}^{D L} \mathbf{v}_{j}^{D L}\right|^{2}, \forall j ; \\
& \text { (C.3) } \sum_{j=1}^{J}\left\|\mathbf{v}_{j}^{D L}\right\|^{2} \leq P_{B S, \max } \text {; } \\
& \text { (C.4) } \sum_{k=1}^{K} P_{k}^{U L} \leq P_{U L, \max } \text {; } \\
& \text { (C.5) } \sum_{r=1}^{R}\left|\mathbf{g}_{R B, r} \sum_{j=1}^{J} \mathbf{v}_{j}^{D L} s_{j}^{D L}\right|^{2} \leq \Psi_{D L} ; \\
& \text { (C.6) } \sum_{k=1}^{K} P_{k}^{U L}\left\|\mathbf{w}_{k}^{U L}\right\|^{2} \leq \Psi_{U L} .
\end{aligned}
$$

However, due to the constraints $(C .1)$ and (C.2), the joint optimization of transmit beamforming at the BS for DL users and power allocation for UL users still becomes intractable. Therefore, we propose an iterative algorithm for attaining the optimal solution in the following section.

Proposition 1: When $R_{R}=R_{T}=R \gg M_{0}$ and $R_{R}=$ $R_{T}=R \gg M_{k}, \forall k$, the interference from cellular system towards the MIMO radar tends to zero.

Proof: From (17), we have

$$
\frac{\operatorname{tr}(\boldsymbol{\Omega})}{\operatorname{tr}\left(\mathbf{V} \mathbf{V}^{H}\right)}=\frac{\left\{\begin{array}{c}
\operatorname{tr}\left(\mathbf{G}_{R B} \sum_{j=1}^{J} \mathbf{v}_{j}^{D L}\left(\mathbf{v}_{j}^{D L}\right)^{H} \mathbf{G}_{R B}^{H}\right) \\
+\sum_{k=1}^{K} P_{k}^{U L}\left\|\mathbf{w}_{k}^{U L}\right\|^{2}+R \sigma_{R}^{2}
\end{array}\right\}}{R^{2}}
$$

$$
\begin{gathered}
=\frac{\operatorname{tr}\left(\mathbf{G}_{R B} \sum_{j=1}^{J} \mathbf{v}_{j}^{D L}\left(\mathbf{v}_{j}^{D L}\right)^{H} \mathbf{G}_{R B}^{H}\right)}{R^{2}} \\
+\frac{\sum_{k=1}^{K} P_{k}^{U L}\left\|\mathbf{w}_{k}^{U L}\right\|^{2}}{R^{2}}+\frac{R \sigma_{R}^{2}}{R^{2}} .
\end{gathered}
$$

When $R \gg M_{0}$ and $R \gg M_{k}$

$$
\lim _{R \rightarrow \infty} \frac{\operatorname{tr}(\boldsymbol{\Omega})}{\operatorname{tr}\left(\mathbf{V} \mathbf{V}^{H}\right)}=0
$$

Remark 1: When $R \rightarrow \infty$, the norm of channels $\mathbf{G}_{R B}$ and $\mathbf{w}_{k}^{U L}$ also increases. However, the increase in the numerator of (21) is limited by constraints (C.5) and (C.6). Hence, when $R$ increases, the norms of channels $\mathbf{G}_{R B}$ and $\mathbf{w}_{k}^{U L}$ will increase to certain values, reach specific limits and stay constant thereafter. However, $R$ in the denominator increases in powers of 2. Hence, when $R \rightarrow \infty$, (21) tends to 0 . Accordingly, when the radar has sufficiently large number of antennas, the detection probability of the radar improves in a spectrum sharing environment. This is because large number of antennas allows for more degrees of freedom which helps in the interference mitigation from the cellular BS and the UL users towards the MIMO radar. This in turn ensures high detection probability, as it is directly proportional to interference mitigation.

\section{Beamforming/Power Optimization Algorithm}

In the section, we propose an iterative algorithm to find the optimal transmit beamformer and power allocation scheme ${ }^{3}$. An alternating minimization approach is adopted to generate an iterative algorithm to obtain a stationary point, e.g., an optimal solution, of the problem (P3). The main optimization problem has two classes of sub-problems: 1) design of transmit beamforming vector $\mathbf{v}^{D L}$ at BS for DL users and 2) design of transmit power vector $\mathbf{p}^{U L}$ for the UL users.

1) Transmit Beamformer $\mathbf{v}^{D L}$ : The beamforming vectors in the problem (P3) appear in terms of the norm square expression. Hence, it is possible to neglect the phase without affecting the optimal solution. Without loss of generality, we confine that the matched output between the channel response $\mathbf{h}_{j}^{D L}$ and the beamforming weight $\mathbf{v}^{D L}$ for each DL user is merely a non-negative real value, i.e., producing the amplitude gain. Thus, when $\mathbf{p}^{U L}$ is fixed, we can find simultaneously $\mathbf{v}^{D L}$ by solving the optimization problem formed using (20a)$(20 \mathrm{~g})$ as follows:

$$
\begin{aligned}
& \text { (P4) } \min _{\mathbf{v}^{D L}, \Psi_{D L}} \Psi_{D L} \\
& \text { subject to }(C .1)-(C .3) \text { and }(C .5) ; \\
& (C .7) \mathfrak{R e}\left(\mathbf{h}_{j}^{D L} \mathbf{v}_{j}^{D L}\right) \geqslant 0, \forall j ; \\
& (C .8) \quad \mathfrak{I m}\left(\mathbf{h}_{j}^{D L} \mathbf{v}_{j}^{D L}\right)=0, \quad \forall j .
\end{aligned}
$$

Lemma 1: The constraint (C.2) can be expressed as a second-order cone convex constraint.

Proof: We can observe that an arbitrary phase rotation can be added to the beamforming vectors $\mathbf{v}_{j}^{D L}$ without affecting the SINR. Therefore, without the loss of generality, $\mathbf{h}_{j}^{D L} \mathbf{v}_{j}^{D L}$

${ }^{3}$ Since the original problem (P1) is non-convex, the optimal transmit beamforming vector and power are referred to as a local maximizer here. 
can be selected to be real. Suppose $\mathbf{V}^{D L}=\left[\mathbf{v}_{l}^{D L}, \ldots, \mathbf{v}_{J}^{D L}\right]$. The SINR constraint (C.2) becomes

$$
\left(1+\frac{1}{\Gamma_{j, \text { min }}^{D L}}\right)\left|\mathbf{h}_{j}^{D L} \mathbf{v}_{j}^{D L}\right|^{2} \geq\left\|{ }_{X}^{\mathbf{h}_{j}^{D L} \mathbf{V}^{D L}}\right\|^{2} ; \quad \forall j,
$$

where $X=\Gamma_{j, \min }^{D L}\left(\sum_{k=1}^{K} P_{k}^{U L}\left|h_{j k}^{D U}\right|^{2}+P_{R}\left\|\mathbf{w}_{j}^{D L}\right\|^{2}+\sigma_{j}^{2}\right)$. Since $\mathbf{h}_{j}^{D L} \mathbf{v}_{j}^{D L}$ can be assumed to be real, we can take the square root of (24). Thus, the constraint $(C .2)$ becomes a second-order cone constraint, which is convex.

Remark 2: Note that the constraints (C.1), (C.3), and $(C .5)$ in (23) are convex. The constraints $(C .7)$ and $(C .8)$ are linear. We can further express $(C .2)$ in (23) as a second-order cone convex constraint using Lemma 1 . Hence, the objective function and all the constraints presented in (P4) are convex with respect to the variables $\mathbf{v}^{D L}$ and $\Psi_{D L}$, and so we can obtain the optimal beamforming weight $\mathbf{v}_{j}^{D L}, \forall j$.

Now, we provide the following lemmas to assist the optimal beamformer and power allocation design.

Lemma 2: If $f$ is a real function of a complex vector $\mathbf{z}$, then the complex gradient vector is given by [49]

$$
\nabla f(\mathbf{z})=\frac{\partial f(\mathbf{z})}{\partial \mathfrak{R e z}}+i \frac{\partial f(\mathbf{z})}{\partial \mathfrak{I m} \mathbf{z}} .
$$

Lemma 3: Assume $f(\mathbf{X})=\left(\mathbf{A X B X}^{T} \mathbf{C}\right)$, where $\mathbf{X}, \mathbf{A}, \mathbf{B}$, and $\mathbf{C}$ are matrices and $\mathbf{A}, \mathbf{B}, \mathbf{C}$ are not a function of $\mathbf{X}$, $\nabla \mathbf{x} \operatorname{Tr}(f(\mathbf{X}))$ can be given as [49]

$$
\frac{\partial \operatorname{Tr}\left(\mathbf{A X B \mathbf { X } ^ { T } \mathbf { C } )}\right.}{\partial \mathbf{X}}=\mathbf{B} \mathbf{X}^{T} \mathbf{C A}+\mathbf{B}^{T} \mathbf{X}^{T} \mathbf{A}^{T} \mathbf{C}^{T} .
$$

Proposition 2: The beamforming weight $\mathbf{v}_{j}^{D L^{H}}$ in the $(t+$ 1)-th iteration can be given as

$\mathbf{v}_{j}^{D L^{H}}(t+1)=$

$\frac{\left(\nu_{j}+\bar{\nu}_{j}\right) J \mathbf{h}_{j}^{D L}}{2\left(\eta+\lambda_{k} K \mathbf{H}_{0}^{H} \mathbf{H}_{0}-\mu_{j} \frac{1}{\Gamma_{j, \text { min }}^{D L}} \mathbf{h}_{j}^{D L} \mathbf{h}_{j}^{D L^{H}}+\kappa \mathbf{G}_{R B} \mathbf{G}_{R B}^{H}\right)}$

Proof: Using Lemma 2 and 3 and by applying KarushKuhn-Tucker (KKT) optimality conditions [48], the partial derivative of the Lagrangian dual function of (23) with respect to $\mathbf{v}_{j}^{D L}$ yields

$$
\begin{aligned}
& 2 \lambda_{k} K \mathbf{v}_{j}^{D L^{H}} \mathbf{H}_{0}^{H} \mathbf{H}_{0}-2 \mu_{j} \frac{1}{\Gamma_{j, m i n}^{D L}} \mathbf{v}_{j}^{D L^{H}} \mathbf{h}_{j}^{D L} \mathbf{h}_{j}^{D L^{H}} \\
& \quad+2 \eta \mathbf{v}_{j}^{D L^{H}}+2 \kappa \mathbf{v}_{j}^{D L^{H}} \mathbf{G}_{R B} \mathbf{G}_{R B}^{H}-\left(\nu_{j}+\bar{\nu}_{j}\right) J \mathbf{h}_{j}^{D L}=0 ; \\
& \Rightarrow \mathbf{v}_{j}^{D L^{H}}(t+1)= \\
& \frac{\left(\nu_{j}+\bar{\nu}_{j}\right) J \mathbf{h}_{j}^{D L}}{2\left(\eta+\lambda_{k} K \mathbf{H}_{0}^{H} \mathbf{H}_{0}-\mu_{j} \frac{1}{\Gamma_{j, m i n}^{D L}} \mathbf{h}_{j}^{D L} \mathbf{h}_{j}^{D L^{H}}+\kappa \mathbf{G}_{R B} \mathbf{G}_{R B}^{H}\right)} .
\end{aligned}
$$

where $\lambda_{k}, \mu_{j}, \eta, \kappa, \nu_{j}$, and $\bar{\nu}_{j}$ are the Lagrangian multipliers associated with the constraints $(C .1)-(C .3),(C .5),(C .7)$, and $(C .8)$, respectively. It can be observed that the update of $\mathbf{v}_{j}^{D L^{H^{*}}}$ depends not only on the Lagrangian multipliers, but also on interference powers.

The Lagrangian multipliers $\lambda_{k}, \mu_{j}, \eta, \kappa, \nu_{j}$, and $\bar{\nu}_{j}$ can be updated using the subgradient method [48]. For example: $\lambda_{k}$ can be updated as follows:
TABLE I: Iterative algorithm for designing $\mathbf{v}^{D L}$ and $\mathbf{p}^{U L}$

$$
\begin{aligned}
& \text { 1: Set the maximum number of iterations } I_{\max } \text {; } \\
& \text { 2: Initialize the iteration counter } k=0 \text {; } \\
& \text { 3: repeat } \\
& \text { 4: } \quad \text { Update } \mathbf{v}^{D L} \text { using (28); } \\
& \text { 5: } \quad \text { Update } \mathbf{p}^{U L} \text { using (31); } \\
& \text { 6: until convergence or } k>I_{\max } \text {. } \\
& \lambda_{k}(t+1)=\left[\lambda_{k}(t)-\varepsilon(t)\left(\sum_{i=1, i \neq k}^{K} P_{i}^{U L^{\star}}(t)\left\|\mathbf{h}_{i}^{U L}\right\|^{2}\right.\right. \\
& +\sum_{j=1}^{J}\left\|\mathbf{H}_{0} \mathbf{v}_{j}^{D L^{\star}}(t)\right\|^{2}+P_{R}\left\|\mathbf{G}_{B R}\right\|^{2} \\
& \left.\left.+N_{0} \sigma_{0}^{2}-\frac{1}{\Gamma_{k, \min }^{U L}} P_{k}^{U L^{\star}}(t)\left\|\mathbf{h}_{k}^{U L}\right\|^{2}\right)\right]^{+},
\end{aligned}
$$

where where $\varepsilon$ is the positive step size. Similarly, we can also update $\mu_{j}, \eta, \kappa, \nu_{j}$, and $\bar{\nu}_{j}$.

2) Transmit power allocation $\mathbf{p}^{U L}$ : For a given $\mathbf{v}^{D L}$, we aim to determine $\mathbf{p}^{U L}$ that maximizes the detection probability of the MIMO radar by minimizing the total interference. Accordingly, we obtain the single-variable optimization problem for designing $\mathbf{p}^{U L}$ from (20a)-(20g) as

$$
\begin{aligned}
& \text { (P5) } \min _{\mathbf{p}^{U L}, \Psi_{U L}} \Psi_{U L} \\
& \text { subject to }(C .1),(C .2),(C .4) \text {, and }(C .6) .
\end{aligned}
$$

Remark 3: The objective function and all the constraints presented in (P5) are convex with respect to $\mathbf{p}^{U L}$ and $\Psi_{U L}$, and thus we can find the optimal power $P_{k}^{U L}, \forall k$.

The $k$-th UL user's power at the $(t+1)$-th iteration, $P_{k}^{U L}(t+$ $1)$, can be updated through the subgradient method [48] as

$$
\begin{aligned}
P_{k}^{U L}(t+1)= & {\left[P_{k}^{U L}(t)-\hat{\varepsilon}(t)\left(\bar{\eta}-\frac{\lambda_{k}}{\Gamma_{k, \text { min }}^{U L}}\left\|\mathbf{h}_{k}^{U L}\right\|^{2}\right.\right.} \\
& \left.\left.+\mu_{k}\left|h_{j k}^{D U}\right|^{2}+\bar{\kappa}\left\|\mathbf{w}_{k}^{U L}\right\|^{2}\right)\right]^{+}, \forall k,
\end{aligned}
$$

where $\hat{\varepsilon}(t)$ is the positive step size in the $t$-th iteration and $\bar{\eta}$, and $\bar{\kappa}$ are the Lagrangian multipliers corresponding to the constraints $(C .4)$, and $(C .6)$, respectively.

The proposed iterative algorithm for designing $\mathbf{v}^{D L}$ and $\mathbf{p}^{U L}$ is described in Table I. The maximum number of iterations is set as $I_{\max }=10$ with initial counter $k=0$. The beamforming weight vector $\mathbf{v}^{D L}$ is updated using (28), while (31) is used to update $\mathbf{p}^{U L}$. We repeat this procedure until the convergence or the iteration counter reaches to $I_{\max }$.

\section{Analysis of Optimal Solutions}

In this section, to gain more insights into the transmit beamforming design and power allocation problem, we analyze the behavior of the optimal solutions in the following different regimes, namely: 1) an interference-dominated regime and 2) a noise-dominated regime.

\section{A. Interference-Dominated Regime (IDR)}

1) Radar IDR: In radar IDR, it is assumed that the interference power generated by radar at the BS and the $j$-th DL user is relatively stronger than the other interference plus noise power, i.e., $P_{R}\left\|\mathbf{G}_{B R}\right\|^{2} \gg$ $\sum_{i=1, i \neq k}^{K} P_{i}^{U L}\left\|\mathbf{h}_{i}^{U L}\right\|^{2}+\sum_{j=1}^{J}\left\|\mathbf{H}_{0} \mathbf{v}_{j}^{D L}\right\|^{2}+N_{0} \sigma_{0}^{2}$ and $P_{R} \|$ 
$\mathbf{w}_{j}^{D L} \|^{2} \gg \sum_{l=1, l \neq j}^{J}\left|\mathbf{h}_{l}^{D L} \mathbf{v}_{l}^{D L}\right|^{2}+\sum_{k=1}^{K} P_{k}^{U L}\left|h_{j k}^{D U}\right|^{2}+\sigma_{j}^{2}$. This occurs particularly when MIMO radar operates at high SNR. Therefore, the problem under this regime is written as

(IDR-P1)

$$
\min _{\mathbf{v}^{D L}, \mathbf{p}^{U L}, \Psi_{U L}, \Psi_{D L}} \Psi_{U L}+\Psi_{D L}
$$

subject to

$$
\begin{aligned}
& \text { (C.1) } \quad P_{R}\left\|\mathbf{G}_{B R}\right\|^{2} \leq \frac{1}{\Gamma_{k, \text { min }}^{U L}} P_{k}^{U L}\left\|\mathbf{h}_{k}^{U L}\right\|^{2}, \forall k ; \\
& \text { (C.2) } \quad P_{R}\left\|\mathbf{w}_{j}^{D L}\right\|^{2} \leq \frac{1}{\Gamma_{j, \text { min }}^{D L}}\left|\mathbf{h}_{j}^{D L} \mathbf{v}_{j}^{D L}\right|^{2}, \quad \forall j ; \\
& \text { (C.3) - (C.8). }
\end{aligned}
$$

Proposition 3: In radar IDR, the optimal transmit beamforming weight $\mathbf{v}_{j}^{D L^{*}}$ at the BS for DL users and power allocation $P_{k}^{U L^{\star}}$ for UL users are given by $\mathbf{v}_{j}^{D L^{H^{\star}}}(t+1)=$

$$
\begin{gathered}
2\left(\eta-\mu_{j} \frac{1}{\Gamma_{j, m i n}^{D L}} \mathbf{h}_{j}^{D L} \mathbf{h}_{j}^{D L^{H}}+\kappa \mathbf{G}_{R B} \mathbf{G}_{R B}^{H}\right) \\
P_{k}^{U L^{\star}}(t+1)=\left[P_{k}^{U L}(t)-\varepsilon(t)\left(\bar{\eta}-\frac{\lambda_{k}}{\Gamma_{k, m i n}^{U L}}\left\|\mathbf{h}_{k}^{U L}\right\|^{2}\right.\right. \\
\left.\left.+\bar{\kappa}\left\|\mathbf{w}_{k}^{U L}\right\|^{2}\right)\right]^{+} ; \quad \forall k . \quad
\end{gathered}
$$

Proof: From Lemma 2 and 3 and using KKT optimality conditions [48], the partial derivative of the Lagrangian dual function of the problem (IDR-P1) with respect to $\mathbf{v}_{j}^{D L}$ yields

$$
\begin{aligned}
- & 2 \mu_{j} \frac{1}{\Gamma_{j, \text { min }}^{D L}} \mathbf{v}_{j}^{D L^{H}} \mathbf{h}_{j}^{D L} \mathbf{h}_{j}^{D L^{H}}+2 \eta \mathbf{v}_{j}^{D L^{H}} \\
& +2 \kappa \mathbf{v}_{j}^{D L^{H}} \mathbf{G}_{R B} \mathbf{G}_{R B}^{H}-\left(\nu_{j}+\bar{\nu}_{j}\right) J \mathbf{h}_{j}^{D L}=0 ; \\
\Rightarrow & \mathbf{v}_{j}^{D L^{H^{*}}}(t+1)= \\
& \left.\frac{\left(\nu_{j}+\bar{\nu}_{j}\right) J \mathbf{h}_{j}^{D L}}{2\left(\eta-\mu_{j} \frac{1}{\Gamma_{j, \text { min }}^{D L}} \mathbf{h}_{j}^{D L} \mathbf{h}_{j}^{D L^{H}}+\kappa \mathbf{G}_{R B} \mathbf{G}_{R B}^{H}\right)} ; \forall j \cdot \quad 35\right)
\end{aligned}
$$

Since the cellular system utilizes the spectrum of the MIMO radar, the $\mathrm{BS}$ are required to generate the minimum interference power towards the MIMO radar so that the detection probability of radar should not hinder. This can be reflected in (35), where the design of the optimal precoder weight vectors $\mathbf{v}_{j}^{D L^{H^{\star}}}$ in the $(t+1)$-th iteration depends not only on the Lagrangian multipliers, but also on interference power generated towards the MIMO radar.

By taking the partial derivative of the Lagrangian dual function of the problem (IDR-P1) with respect to $P_{k}^{U L}$ and equating the result to zero, the power update for the $k$-th UL user's at the $(t+1)$-th iteration can be expressed as

$$
\begin{array}{r}
P_{k}^{U L^{\star}}(t+1)=\left[P_{k}^{U L}(t)-\varepsilon(t)\left(\bar{\eta}-\frac{\lambda_{k}}{\Gamma_{k, \text { min }}^{U L}}\left\|\mathbf{h}_{k}^{U L}\right\|^{2}\right.\right. \\
\left.\left.+\bar{\kappa}\left\|\mathbf{w}_{k}^{U L}\right\|^{2}\right)\right]^{+} ; \quad \forall k
\end{array}
$$

2) Multiuser IDR: In multiuser IDR, it is assumed that the interference power generated by UL users at the BS for $k$-th UL user and the interference power due to DL users at the $j$-th DL user are relatively stronger than the other interference terms plus noise power, i.e., $\sum_{i=1, i \neq k}^{K} P_{i}^{U L}\left\|\mathbf{h}_{i}^{U L}\right\|^{2} \gg \sum_{j=1}^{J} \|$ $\mathbf{H}_{0} \mathbf{v}_{j}^{D L}\left\|^{2}+P_{R}\right\| \mathbf{G}_{B R} \|^{2}+N_{0} \sigma_{0}^{2}$ and $\sum_{l=1, l \neq j}^{J}\left|\mathbf{h}_{l}^{D L} \mathbf{v}_{l}^{D L}\right|^{2} \gg$ $\sum_{k=1}^{K} P_{k}^{U L}\left|h_{j k}^{D U}\right|^{2}+P_{R}\left\|\mathbf{w}_{j}^{D L}\right\|^{2}+\sigma_{j}^{2}$. Thus, the optimization problem under this regime can be formulated as

(IDR-P2) $\min _{\mathbf{v}^{D L}, \mathbf{p}^{U L}, \Psi_{U L}, \Psi_{D L}} \Psi_{U L}+\Psi_{D L}$

subject to

(C.1) $\sum_{i=1, i \neq k}^{K} P_{i}^{U L}\left\|\mathbf{h}_{i}^{U L}\right\|^{2} \leq \frac{1}{\Gamma_{k, \text { min }}^{U L}} P_{k}^{U L}\left\|\mathbf{h}_{k}^{U L}\right\|^{2}, \quad \forall k ;$

(C.2) $\sum_{l=1, l \neq j}^{J}\left|\mathbf{h}_{l}^{D L} \mathbf{v}_{l}^{D L}\right|^{2} \leq \frac{1}{\Gamma_{j, \text { min }}^{D L}}\left|\mathbf{h}_{j}^{D L} \mathbf{v}_{j}^{D L}\right|^{2}, \forall j$

$(C .3)-(C .8)$,

Proposition 4: In multiuser IDR, the expression for the optimal transmit beamforming $\mathbf{v}_{j}^{D L^{H^{\star}}}$ is given by

$$
\frac{\mathbf{v}_{j}^{D L^{H^{\star}}}(t+1)=}{2\left(\eta-\mu_{j} \frac{1}{\Gamma_{j, \text { min }}^{D L}} \mathbf{h}_{j}^{D L} \mathbf{h}_{j}^{D L^{H}}+\kappa \mathbf{G}_{R B} \mathbf{G}_{R B}^{H}\right)} ; \forall j
$$

where the optimal transmit power $P_{k}^{U L}$ for $k$-th UL user is written as

$$
\begin{array}{r}
P_{k}^{U L^{\star}}(t+1)=\left[P_{k}^{U L}(t)-\varepsilon(t)\left(\bar{\eta}-\frac{\lambda_{k}}{\Gamma_{k, \text { min }}^{U L}}\left\|\mathbf{h}_{k}^{U L}\right\|^{2}\right.\right. \\
\left.\left.+\bar{\kappa}\left\|\mathbf{w}_{k}^{U L}\right\|^{2}\right)\right]^{+} ; \forall k .
\end{array}
$$

Proof: Applying KKT conditions [48] and using Lemma 2 and 3 , we have

$$
\begin{aligned}
& -2 \mu_{j} \frac{1}{\Gamma_{j, \text { min }}^{D L}} \mathbf{v}_{j}^{D L^{H}} \mathbf{h}_{j}^{D L} \mathbf{h}_{j}^{D L^{H}}+2 \eta \mathbf{v}_{j}^{D L^{H}} \\
& \quad+2 \kappa \mathbf{v}_{j}^{D L^{H}} \mathbf{G}_{R B} \mathbf{G}_{R B}^{H}-\left(\nu_{j}+\bar{\nu}_{j}\right) J \mathbf{h}_{j}^{D L}=0 ; \\
& \Rightarrow \mathbf{v}_{j}^{D L^{H^{\star}}}(t+1)= \\
& \frac{\left(\nu_{j}+\bar{\nu}_{j}\right) J \mathbf{h}_{j}^{D L}}{2\left(\eta-\mu_{j} \frac{1}{\Gamma_{j, \text { min }}^{D L}} \mathbf{h}_{j}^{D L} \mathbf{h}_{j}^{D L^{H}}+\kappa \mathbf{G}_{R B} \mathbf{G}_{R B}^{H}\right)} ; \forall j
\end{aligned}
$$

The power update for $k$-th UL user at the $(t+1)$-th iteration is expressed as

$$
\begin{array}{r}
P_{k}^{U L^{\star}}(t+1)=\left[P_{k}^{U L}(t)-\right. \\
-\varepsilon(t)\left(\bar{\eta}-\frac{\lambda_{k}}{\Gamma_{k, \text { min }}^{U L}}\left\|\mathbf{h}_{k}^{U L}\right\|^{2}\right. \\
\left.\left.+\bar{\kappa}\left\|\mathbf{w}_{k}^{U L}\right\|^{2}\right)\right]^{+} ; \forall k . \quad
\end{array}
$$


The update of $\mathbf{v}_{j}^{D L^{H^{\star}}}$ and $P_{k}^{U L^{\star}}$ are identical to (35) and (36).

\section{B. BS Noise-Dominated Regime (BS-NDR)}

Now, we turn to BS-NDR, in which the BS noise is much stronger than the interference terms, i.e., $N_{0} \sigma_{0}^{2} \gg$ $\sum_{i=1, i \neq k}^{K} P_{i}^{U L}\left\|\mathbf{h}_{i}^{U L}\right\|^{2}+\sum_{j=1}^{J}\left\|\mathbf{H}_{0} \mathbf{v}_{j}^{D L}\right\|^{2}+P_{R}\left\|\mathbf{G}_{B R}\right\|^{2}$ In this scenario, the FD BS operates at high SNR and thus, the optimization problem is formulated as

(NDR-P1) $\min _{\mathbf{v}^{D L}, \mathbf{p}^{U L}, \Psi_{U L}, \Psi_{D L}} \Psi_{U L}+\Psi_{D L}$

subject to

$$
\begin{aligned}
& \text { (C.1) } N_{0} \sigma_{0}^{2} \leq \frac{1}{\Gamma_{k, \text { min }}^{U L}} P_{k}^{U L}\left\|\mathbf{h}_{k}^{U L}\right\|^{2}, \quad \forall k ; \\
& \text { (C.2) } \sum_{l=1, l \neq j}^{J}\left|\mathbf{h}_{l}^{D L} \mathbf{v}_{l}^{D L}\right|^{2}+\sum_{k=1}^{K} P_{k}^{U L}\left|h_{j k}^{D U}\right|^{2} \\
& \quad+P_{R}\left\|\mathbf{w}_{j}^{D L}\right\|^{2}+\sigma_{j}^{2} \leq \frac{1}{\Gamma_{j, \text { min }}^{D L}}\left|\mathbf{h}_{j}^{D L} \mathbf{v}_{j}^{D L}\right|^{2}, \forall j ; \\
& (C .3)-(C .8) .
\end{aligned}
$$

In this regime, the update equations of the beamforming for UL users and transmit power for DL users are written as:

$$
\begin{aligned}
& \frac{\mathbf{v}_{j}^{D L^{H^{*}}}(t+1)=}{2\left(\eta-\mu_{j} \frac{1}{\Gamma_{j, \text { min }}^{D L}} \mathbf{h}_{j}^{D L} \mathbf{h}_{j}^{D L^{H}}+\kappa \mathbf{G}_{R B} \mathbf{G}_{R B}^{H}\right)} ; \quad \forall j, \quad \text { (4) } \\
& P_{k}^{U L^{\star}}(t+1)=\left[P_{k}^{U L}(t)-\varepsilon(t)\left(\bar{\eta}-\frac{\lambda_{k}}{\Gamma_{k, \text { min }}^{U L}}\left\|\mathbf{h}_{k}^{U L}\right\|^{2}\right.\right. \\
& \left.\left.+\mu_{k}\left|h_{j k}^{D U}\right|^{2}+\bar{\kappa}\left\|\mathbf{w}_{k}^{U L}\right\|^{2}\right)\right]^{+} ; \forall k
\end{aligned}
$$

\section{Complexity ANALYSiS}

The main computation complexity of the designed iterative algorithm in Table I depends on solving the SOCP problem (23). A real-valued SOCP problem can be expressed as

$$
\begin{array}{cl}
\min _{\mathbf{x} \in \mathcal{R}^{n}} & \mathbf{c}^{T} \mathbf{x} \\
\text { s.t. } & \left\|\mathbf{A}_{i} \mathbf{x}+\mathbf{b}_{i}\right\| \leq \mathbf{c}_{i}^{T} \mathbf{x}+d_{i}, i=1, \ldots, P, \\
& \|\mathbf{x}\|_{2} \leq R,
\end{array}
$$

where $\mathbf{A}_{i}$ denotes the symmetric block-diagonal matrices with $Q$ diagonal blocks of size $a_{l} \times a_{l}$, where $l=1,2, \ldots, Q$, and $\mathbf{b}_{i} \in \mathcal{R}^{a_{i}}$. As discussed in [50], the upper bound on the number of required arithmetic operations to solve this problem is $\mathcal{O}(1)(1+Q)^{1 / 2} n\left(n^{2}+Q+\sum_{i=0}^{Q} a_{i}^{2}\right)$. In our problem, the number of inequalities $Q+1$ equals to $K+J+4$. For the SINR constraint of each UL user, $a_{i}=4 N_{0}^{2}+1, \forall i \in$ $\{1,2, \ldots K\}$. Similarly, for the DL user's SINR constraint, $a_{i}=4, \forall i \in\{1,2, \ldots K\}$. For the BS power constraint, the dimension of the block is $a_{i}=M_{0}^{2} \forall i \in\{1,2, \ldots K\}$, and for UL user power constraint $a_{i}=1, \forall i \in\{1,2, \ldots K\}$, whereas for the MIMO radar interference constraint, the dimension of the blocks are $a_{r}=1, \forall r \in\{1,2, \ldots, R\}$. The dimension of

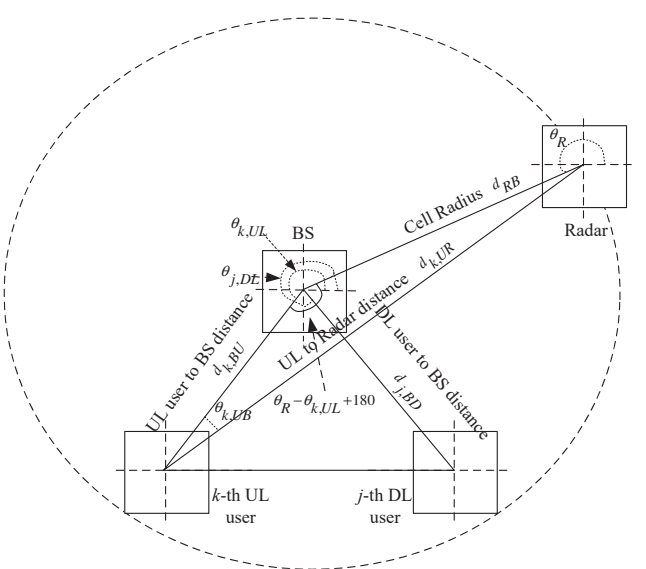

Fig. 2: Geographical locations of an FD MIMO communication system with spectrum sharing MIMO radar.

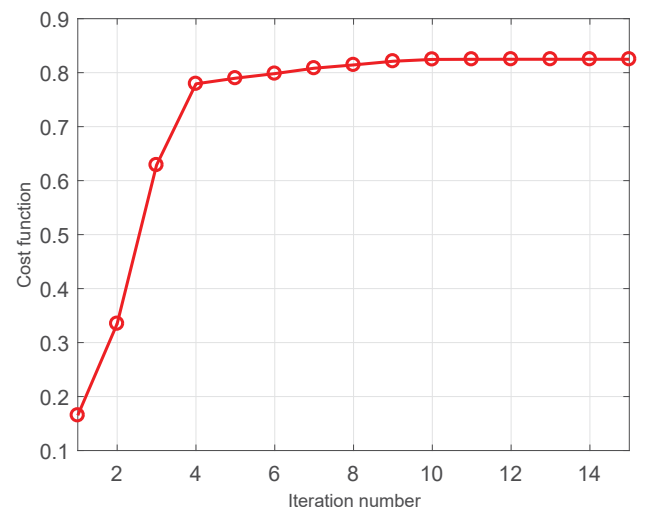

Fig. 3: Convergence behavior of the proposed iterative algorithm.

the blocks due to the constraint $(C .6)$ is $a=R_{R}^{2}$. The size of the unknown variables is $n=2 J M_{0}+1$. The complexity due to the subproblem (30) is $K\left(4 N_{0}^{2}+1\right)+4 J+K+K R_{R}^{2}$.

\section{NUMERICAL RESULTS}

In this section, we provide simulation results to quantify the performance of the proposed iterative algorithm for spectrum sharing. It is assumed that all the channel links are complex Gaussian distribution and independent and identically distributed (i.i.d.). However, for the self-interference channel, we adopt the Rician model in [18]. In particular, the self-interference channel is distributed as $\tilde{\mathbf{H}}_{0} \sim$ $\mathcal{C N}\left(\sqrt{\frac{K_{R}}{1+K_{R}}} \hat{\mathbf{H}}_{0}, \frac{1}{1+K_{R}} \mathbf{I}_{N_{0}} \otimes \mathbf{I}_{M_{0}}\right)$, where $K_{R}$ is the Rician factor, and $\hat{\mathbf{H}}_{0}$ is a deterministic matrix ${ }^{4}$.

The total number of UL and DL users are set to four, i.e., $K=J=4$. The BS transmit power is set at $44 \mathrm{dBm}$, while for the UL users it is set at $30 \mathrm{dBm}$. To model the spectrum sharing network, we consider a Cartesian coordinate system with $X$ and $Y$ axes as shown in Fig. 2. The path-loss exponent $\alpha$ is set at 4 , while the carrier-frequency under consideration is $3.6 \mathrm{GHz}$. It is worth noting that the proposed model is not limited to this frequency. The $3.6 \mathrm{GHz}$ carrier frequency is considered just for reference. The proposed model can also be utilized in other frequency bands $(2-4 \mathrm{GHz}$ in the $\mathrm{UK}$, $2.3-2.4 \mathrm{GHz}$ in Europe, etc.) around the world, albeit certain

${ }^{4}$ Without loss of generality, we set $K_{R}=1$ and $\tilde{\mathbf{H}}_{0}$ to be the matrix of all ones for all the simulations as was considered in [25]. 


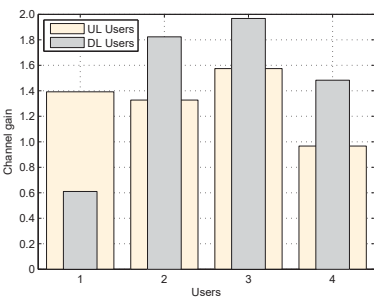

(a) Channel gain versus Users

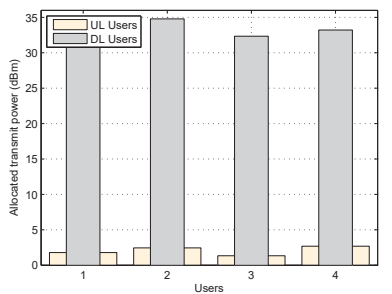

(b) Power allocation in Radar IDR

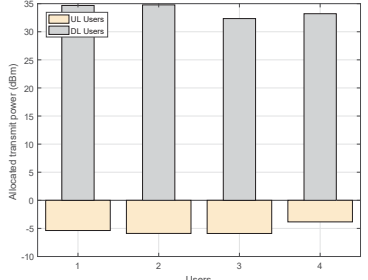

(c) Power allocation in MU IDR

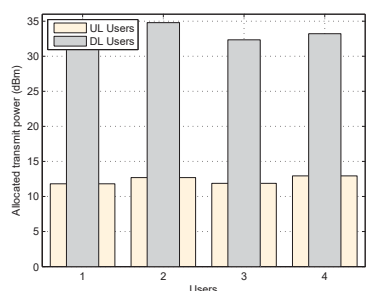

(d) Power allocation in BS NDR

Fig. 4: An example of power allocation under fixed channel gains in different operating regimes.

changes, such frequency dependent path loss and line of sight propagation parameters.

A MIMO radar is placed at the circumference of a circular cell, with cell radius $d_{R B}=40$ meters (m). The BS is fixed at the centre of the cell, whereas the UL user and DL users are placed randomly in the cell. The position of each UL and DL user with respect to the BS can be given by $d_{k, B U}$ and $d_{j, B D}$, and the angle between the BS-UL-Radar is $\theta_{k, B U}$. Similarly, the angle between radar-BS-UL is given by $\theta_{R}-\theta_{k, U L}+180$. Thus the distance between the $k$-th UL user and radar can be given by using law of cosines as

$$
\begin{aligned}
& d_{k, U R}= \\
& \sqrt{d_{k, B U}^{2}+d_{R B}^{2}-2 d_{k, B U} d_{R B} \cos \left(\theta_{R}-\theta_{k, U L}+180\right)} .
\end{aligned}
$$

Further by using law of sines, it can be shown that

$$
\theta_{k, B U}=\sin ^{-1}\left(\frac{d_{R B}}{d_{k, U R}} \sin \left(\theta_{R}-\theta_{k, U L}+180\right)\right) .
$$

Moreover, the angle between the $k$-th UL and $j$-th DL user can be expressed as

$$
\theta_{j, k, D U}=\left|\theta_{j, D L}-\theta_{k, U L}\right| .
$$

Unless otherwise stated, we assume $R_{T}=R_{R}=4$, $M_{0}=N_{0}=N=2, \theta_{R} \in\left\{180^{\circ}, \ldots, 270^{\circ}\right\}, \theta_{k, U L} \in$ $\left\{180^{\circ}, \ldots, 240^{\circ}\right\}, \theta_{k, D L} \in\left\{270^{\circ}, \ldots, 330^{\circ}\right\}, P_{F A}=10^{-4}$, $\Gamma_{k, \text { min }}^{U L}=2 \mathrm{~dB}, \forall k$ and $\Gamma_{j, \text { min }}^{U L}=3 \mathrm{~dB}, \forall j$, in the following simulation results.

We begin by illustrating the convergence behaviour of the proposed algorithm for a single channel realization in Fig. 3. In particular, we set the maximum number of iterations to 10 . As can be seen from the figure, the cost function, i.e., the detection probability performance, of the proposed algorithm increases monotonically and converges in less four iterations.

We now show the amount of allocated power in three different regimes with $K=J=4$ and $P_{R}=20 \mathrm{~dB}$ in Fig. 4 . In particular, in Fig. 4(a), the channel gains with path loss for UL users are set as $[1.3918,1.3276,1.5745,0.9668]$, while those for the DL users are [0.6104, 1.8232, 1.9676, 1.4834]. For radar interference-dominated regime in Fig. 4(b), the optimal transmit beamforming design for DL users behaves similar to the water-filling approach, and the water-filling level depends not only on the channel gain, but also on the interference power. The UL user in this regime with a higher channel gain is allocated with less power, while more power is allocated to the UL user with worst channel gain. Fig. 4(c) illustrates the optimal resource allocation policies in the multiuser interference-dominated regime, where the optimal

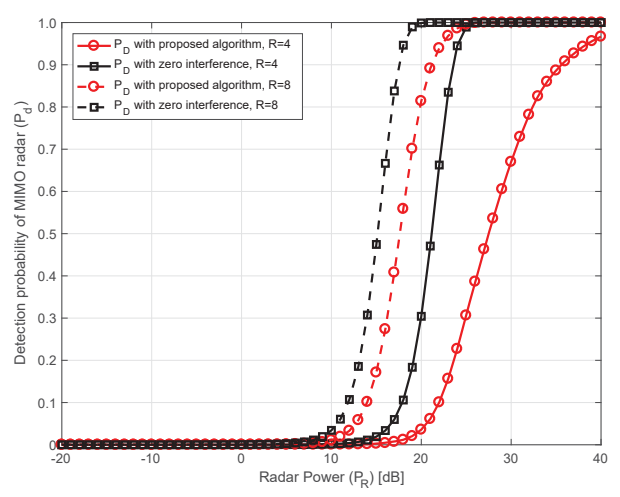

Fig. 5: Detection probability of radar $P_{D}$ versus radar power $P_{R}$.

beamforming for DL users follows as in Fig. 4(b). However, the UL users are allocated with very less power as compared to the DL users due to MU interference powers. As shown in Fig. 4(d), the allocated power for UL users in the BS noisedominated regime depends to UL user's channel gain, and the user with a better channel gain is allocated with less power. It is worth noting that the resource allocation in this regime for DL users is similar to water-filling approach.

Hereinafter, we illustrate the performance of the MIMO radar and the cellular system. In Fig. 5, we show the detection probability of the MIMO radar with respect to radar transmit power. Here, we consider two cases: 1) $R=8$ (dashed lines) and 2) $R=4$ (straight lines). It can be seen that for fixed $P_{F A}$, in order to achieve a particular $P_{D}$ the radar needs more power than the case without spectrum sharing scenario (zero interference). Also it can be seen that the radar needs more power when $R=4$ than $R=8$ to achieve similar performance. Besides, the gap in performance between spectrum sharing and without spectrum sharing cases reduces when $R$ increases. This is because, while the number of antennas at the cellular system (BS and UEs) are fixed, increasing the radar antennas, increases the degree of freedoms of the radar, which ensures a better detection performance for the radar even with spectrum sharing.

Next, in Fig. 6, we plot $P_{D}$ for different values of $P_{F A}$ and $P_{R}=\{15,20\} \mathrm{dB}$. Here, $\Gamma=0 \mathrm{~dB}$. Similar to the previous figure, the detection probability of the radar is better at high $P_{R}$ and small $P_{F A}$ when the radar is not sharing its spectrum. However when $P_{F A}$ is small, detection performance of radar with spectrum sharing is somewhat comparable to the case without spectrum sharing. 


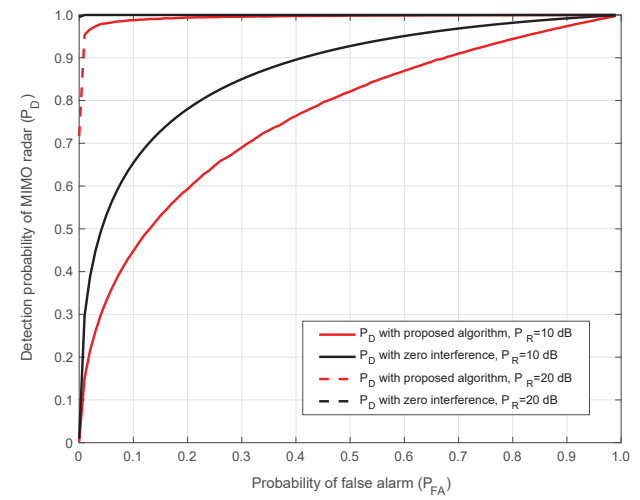

Fig. 6: Detection probability of radar $P_{D}$ versus false alarm $P_{F A}$.

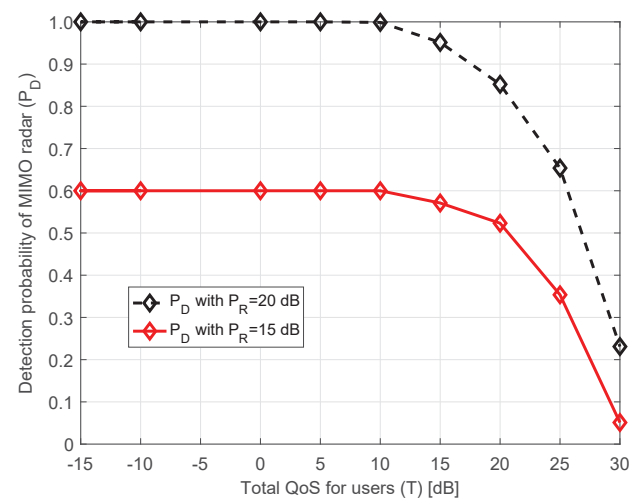

Fig. 7: Detection probability of radar $P_{D}$ versus QoS of cellular user.

Finally, in Fig. 7 we compare the performance of the radar with respect to the total QoS (in terms of SINR) of the users $(\mathrm{UL}+\mathrm{DL})$ in the cellular system. It can be seen that $P_{D}$ decreases as we increase the QoS requirements of the users. This shows the trade-off in performance between both systems. While increasing the QoS requirements will ensure more data rate for the users, it will also induce more interference towards the radar system, which in turn will reduce the detection probability of the radar.

At this point, we would like to note that spectrum sharing comes at a cost, which in this case is paid by the radar. The price here is that the radar requires more power to attain a particular detection probability when sharing its spectrum with the cellular system. While the main beneficiary here is the cellular system, which otherwise will be left void of any connectivity, this is in conjunction to the immediate demand for extra spectrum in commercial communication systems.

\section{CONCLUSION}

The optimization problem for joint design of beamforming weights at the BS and power allocation for UL users were formulated to facilitate the coexistence of a FD cellular system and a MIMO radar when the QoS of each cellular user and the transmit power budget of the BS and UL users are given. Numerical results demonstrated the trade-off in performance between radar and cellular system. In particular, spectrum sharing with a cellular system comes at the price of increasing radar power by up-to $3-4 \mathrm{~dB}$ if the detection probability of the radar is left unaltered.

\section{REFERENCES}

[1] Cisco visual networking index: Global mobile data traffic forecast, 2015-2020. [Online] Available: http://www.cisco.com/c/en/us/solutions/collateral/service-provider/visualnetworking-index-vni/vni-forecast-qa.pdf

[2] Federal Communications Commission (FCC), Spectrum policy task force, [Online] Available: https://transition.fcc.gov/sptf/files/IPWGFinalReport.pdf, Nov. 2002.

[3] K. Singh, S. Biswas, A. Gupta, T. Ratnarajah, and M. Sellathurai, "Joint power allocation and beamforming design for full-duplex MIMO cellular systems with spectrum sharing radar," in Proc. IEEE NASA/ESA Conf. Adaptive Hardware and Sys. (AHS), Jul. 2017, pp. 93-100.

[4] S. Haykin, "Cognitive radio: Brain-empowered wireless communications," IEEE J. Sel. Areas Commun., vol. 23, no. 2, pp. 201-220, Feb. 2005 .

[5] Federal Communications Commission (FCC), Facilitating opportunities for flexible, efficient, and reliable spectrum use employing cognitive radio technologies, [Online] Available: https://apps.fcc.gov/edocs_public /attachmatch/FCC-10-12A1_Rcd.pdf, Jan. 2010.

[6] F. H. Sanders, R. L. Sole, B. L. Bedford, D. Franc, and T. Pawlowitz, "Effects of RF interference on radar receivers," Nat. Telecommun. Inf. Admin, Washington, DC, USA, Tech. Rep. TR-06-444, Sep. 2006.

[7] H. Griffiths, L. Cohen, S. Watts, E. Mokole, C. Baker, M. Wicks, and S. Blunt, "Radar spectrum engineering and management: Technical and regulatory issues," Proc. IEEE, vol. 103, no. 1, pp. 85-102, Jan. 2015.

[8] M. P. Fitz, T. R. Halford, I. Hossain, and S. W. Enserink, "Towards simultaneous radar and spectrum sensing," in Proc. IEEE Int. Symp. Dyn. Spectr. Access Netw. (DYSPAN), Apr. 2014, pp. 15-19.

[9] CEPT, "Licensed Shared Access (LSA)," Electronic Communications Committees (ECC), [Online] Available: http://www.erodocdb.dk/Docs /doc98/official/pdf/ECCREP205.PDF, Feb. 2014.

[10] M. Matinmikko, M. Mustonen, D. Roberson, J. Paavola, M. Hoyhtya, S. Yrjola, and J. Roning, "Overview and comparison of recent spectrum sharing approaches in regulation and research: From opportunistic unlicensed access towards licensed shared access," in Proc. IEEE Int. Symp. Dyn. Spectr. Access Netw. (DYSPAN), Apr. 2014, pp. 92-102.

[11] Federal Communications Commission (FCC), Connecting America: The national broadband plan, [Online] Available: https://www.fcc.gov/general/national-broadband-plan.pdf, Mar. 2010.

[12] President's Council of Advisors on Science and Technology, Realizing the full potential of government-held spectrum to spur economic growth, [Online] Available: https://obamawhitehouse.archives.gov/sites/default /files/microsites/ostp/ pcast_spectrum_report_final_july_20_2012.pdf, Jul. 2012.

[13] Federal Communications Commission (FCC), In the master of revision of parts 2 and 15 of the commission's rules to permit unlicensed national information infrastructure devices in the $5 \mathrm{GHz}$ band, ET Docket No. 03122,2006

[14] National Telecommunications and Information Administration (NTIA), An assessment of the near-term viability of accommodating wireless broadband systems in the 1675-1710 $\mathrm{MHz}, 1755$ $1780 \mathrm{MHz}, \quad 3500-3650 \mathrm{MHz}, \quad 4200-4220 \mathrm{MHz}$, and 4380$4400 \mathrm{MHz}$ bands (Fast track report), [Online] Available: https://www.ntia.doc.gov/files/ntia/publications/fasttrackevaluation_1115 2010.pdf, Nov. 2010.

[15] Federal Communications Commission (FCC), FCC proposes innovative small cell use in $3.5 \mathrm{GHz}$ band, [Online] Available: https://www.fcc.gov/document/fcc-proposes-innovative-small-cell-use35-ghz-band, accessed, Dec. 2012.

[16] J. H. Reed et al., "On the co-existence of TD-LTE and radar over 3.5 GHz band: An experimental study," IEEE Wireless Commun. Lett., vol. 5, no. 4, pp. 368-371, Aug. 2016.

[17] V. Ramaswamy and J. T. Correia, "Enabling spectrum sharing between LTE and RADAR systems in S-band," in Proc. IEEE WCNC, Mar, 2017, pp. 1-6.

[18] M. Duarte, C. Dick, and A. Sabharwal, "Experiment-driven characterization of full-duplex wireless systems," IEEE Trans. Wireless Commun., vol. 11, no. 12, pp. 4296-4307, Dec. 2012.

[19] D. Bharadia and S. Katti, "Full duplex MIMO radios," USENIX NSDI, pp. 359-372, 2014

[20] Y. Hua et al., "Radio self-interference cancellation by transmit beamforming, all-analog cancellation and blind digital tuning," Elsevier Signal Process., vol. 108, pp. 322-340, Mar. 2015.

[21] A. Sahai, G. Patel, C. Dick, and A. Sabharwal, "On the impact of phase noise on active cancelation in wireless full-duplex," IEEE Trans. Vel. Technol., vol. 62, no. 9, pp. 4494-4510, Nov. 2013. 
[22] B. P. Day, A. R. Margetts, D. W. Bliss, and P. Schniter, "Full-duplex bidirectional MIMO: Achievable rates under limited dynamic range," IEEE Trans. Signal Process., vol. 60, no. 7, pp. 3702-3713, Jul. 2012.

[23] W. Li, J. Lilleberg, and K. Rikkinen, "On rate region analysis of half- and full-duplex OFDM communication links," IEEE J. Sel. Areas Commun., vol. 32, no. 9, pp. 1688-1698, Sep. 2014

[24] T. M. Kim, H. J. Yang, and A. Paulraj, "Distributed sum-rate optimization for full-duplex MIMO system under limited dynamic range," IEEE Signal Process. Letters, vol. 20, no. 6, pp. 555-558, Jun. 2013.

[25] D. Nguyen, L. Tran, P. Pirinen, and M. Latva-aho, "On the spectral efficiency of full-duplex small cell wireless systems," IEEE Trans. Wireless Commun., vol. 13, no. 9, pp. 4896-4910, Sep. 2014.

[26] S. Li, R. Murch, and V. Lau, "Linear transceiver design for full-duplex multi-user MIMO system," in Proc. IEEE ICC, pp. 4921-4926, Jun. 2014

[27] M. J. Marcus, "Sharing government with private users: Opportunities and challenges," IEEE Wireless Commun., vol. 16, no. 3, pp. 4-5, Jun. 2009.

[28] F. Hessar and S. Roy, "Spectrum sharing between a surveillance radar and secondary Wi-Fi networks," IEEE Trans. Aerosp. Electron. Syst., vol. 52, no. 3, pp. 1434-1447, Jul. 2016.

[29] S.-S. Raymond, A. Abubakari, and H.-S. Jo, "Coexistence of powercontrolled cellular networks with rotating radar," IEEE J. Sel. Areas Commun., vol. 34, no. 10, pp. 2605-2616, Oct. 2016.

[30] R. Saruthirathanaworakun, J. M. Peha, and L. M. Correia, "Opportunistic sharing between rotating radar and cellular," IEEE J. Sel. Areas Commun., vol. 30, no. 10, pp. 1900-1910, Nov. 2012

[31] R. Saruthirathanaworakun, J. M. Peha, and L. M. Correia, "Performance of data services in cellular networks sharing spectrum with a single rotating radar," in Proc. IEEE WoWMoM, Jun. 2012, pp. 1-6.

[32] J. Li and P. Stoica, "MIMO radar with colocated antennas," IEEE Signal Process. Mag., vol. 24, no. 5, pp. 106-114, Sep. 2007.

[33] J. Li and P. Stoica, MIMO Radar Signal Processing, New York, NY, USA: Wiley, 2008.

[34] A. Babaei, W. H. Tranter, and T. Bose, "A nullspace-based precoder with subspace expansion for radar/communications coexistence," in Proc. IEEE GLOBECOM, Dec. 2013, pp. 3487-3492.

[35] A. Khawar et al., "Target detection performance of spectrum sharing MIMO radar," IEEE Sensors J., vol. 15, no. 9, pp. 4928-4940, Sep. 2015.

[36] A. Khawar et al., "Spectrum Sharing Between S-band Radar and LTE Cellular System: A Spatial Approach,” in Proc. IEEE DYSPAN, Apr. 2014 pp. 7-14.

[37] B. Li, A. Petropulu, and W. Trappe, "Optimum co-design for spectrum sharing between matrix completion based MIMO radars and a MIMO communication system," IEEE Trans. Signal Process., vol. 64, no. 17, pp. $4562-4575$, Sep. 2016

[38] B. Li and A. Petropulu, "MIMO radar and communication spectrum sharing with clutter mitigation," in Proc. IEEE RadarConf, May 2016, pp. 1-6.

[39] H. Deng et al., "Interference mitigation processing for spectrum-sharing between radar and wireless communication systems," IEEE Trans. Aerosp. Electron. Syst., vol. 49, no. 3, pp. 1911-1919, Jul. 2013.

[40] F. Paisana, J. P. Miranda, N. Marchetti, and L. A. Dasilva, "Databaseaided sensing for radar bands," in Proc. IEEE DYSPAN, Apr. 2014, pp. $1-6$

[41] P. Wu, R. Schober, and V. K. Bhargava, "Robust transceiver design for SC-FDE multi-hop full-duplex decode-and-forward relaying systems," IEEE Trans. Wireless Commun., vol. 15, no. 2, pp. 1129-1145, Feb. 2016

[42] S. Huberman and T. Le-Ngoc, "Sequential convex programming for fullduplex single-user MIMO systems," in Proc. IEEE ICC, Jun. 2014, pp. 5078-5082.

[43] Y. Gu, H. Chen, Y. Li, and B. Vucetic, "Ultra-reliable short-packet communications: half-duplex or full-duplex relaying?," IEEE Wireless Commun. Lett., vol. PP, no. 99, pp. 1-1, 2017.

[44] J. Fan et al., "Generalized likelihood ratio statistics and wilks phenomenon," The Ann. Stat., vol. 29, no. 1, pp. 153-193, Feb. 2001

[45] L. Xu and J. Li, "Iterative generalized-likelihood ratio test for MIMO radar," IEEE Trans. Signal Process., vol. 55, no. 6, pp. 2375-2385, Jun. 2007

[46] I. Bekkerman and J. Tabrikian, "Target detection and localization using MIMO radars and sonars," IEEE Trans. Signal Process., vol. 54, no. 10, pp. $3873-3883$, Oct. 2006

[47] S. M. Kay, Fundamentals of statistical signal processing: Detection theory, vol. 2, Prentice Hall, 1998.

[48] S. Boyd and L. Vandenberghe, Convex optimization, Cambridge, U.K. Cambridge University Press, 2004

[49] B. P. Kaare and S. P. Michael, "The Matrix Cookbook," http://matrixcookbook.com, Nov. 14, 2008
[50] A. Ben-Tal and A. Nemirovski, Lectures on Modern Convex Optimization: Analysis, Algorithms, Engineering Applications. SIAM, 2001.

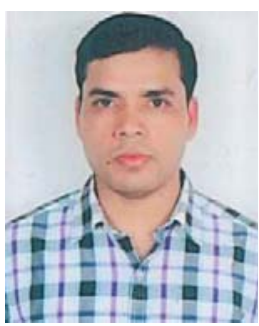

Keshav Singh (S'12, M'16) received the degree of Master of Technology (with first-class honors) in Computer Science from Devi Ahilya Vishwavidyalaya, Indore, India, in 2006, the M.Sc. in Information \& Telecommunications Technologies from Athens Information Technology, Greece, in 2009, and the Ph.D. degree in Communication Engineering from National Central University, Taiwan, in 2015. Since 2016, he has been with Institute for Digital Communications, School of Engineering University of Edinburgh, where he is currently working as a Research Associate. He has also served as a Technical Program Committee Member for numerous IEEE conferences. His current research interests are in the areas of Green Communications, Resource Allocation, FullDuplex Radio, Cooperative and Energy Harvesting Networks, Multiple-Input and Multiple-Output (MIMO), Non-Orthogonal Multiple Access (NOMA), and Wireless Caching.

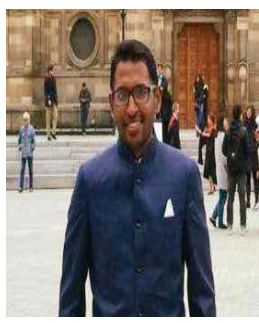

Sudip Biswas (S'16, M'17) received the B.Tech degree in Electronics and Communication Engineering from Sikkim Manipal Institute of Technology, Sikkim, India, in 2010, the M.Sc. degree in Signal Processing and Communications from the University of Edinburgh, Edinburgh,U.K., in 2013, and the Ph.D. degree in Digital Communications from the University of Edinburgh's Institute for Digital Communications (IDCOM) in 2017. Currently, he is working as a Research Scientist in IDCOM University of Edinburgh. His research interests include various topics in wireless communications and network information theory with particular focus on possible $5 \mathrm{G}$ technologies such as Massive Multiple-Input and Multiple-Output (MIMO), mmWave, Full-Duplex Radio, Wireless Caching, Non-Orthogonal Multiple-Access, and Machine Learning for Communications.

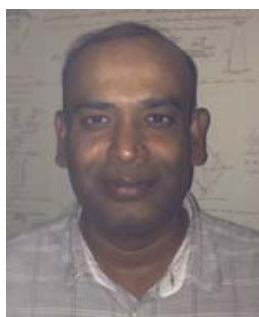

Tharmalingam Ratnarajah (A'96-M'05-SM'05) is currently with the Institute for Digital Communications, University of Edinburgh, Edinburgh, UK, as a Professor in Digital Communications and Signal Processing. His research interests include signal processing and information theoretic aspects of $5 \mathrm{C}$ and beyond wireless networks, full-duplex radio, mmWave communications, random matrices theory, interference alignment, statistical and array signal processing and quantum information theory. He has published over 330 publications in these areas and holds four U.S. patents. He was the coordinator of the FP7 projects ADEL $(3.7 \mathrm{M} €)$ in the area of licensed shared access for $5 \mathrm{G}$ wireless networks and HARP $(4.6 \mathrm{M} €)$ in the area of highly distributed MIMO and FP7 Future and Emerging Technologies projects HIATUS $(3.6 \mathrm{M} €)$ in the area of interference alignment and CROWN (3.4M€) in the area of cognitive radio networks. D Ratnarajah is a Fellow of Higher Education Academy (FHEA), U.K..

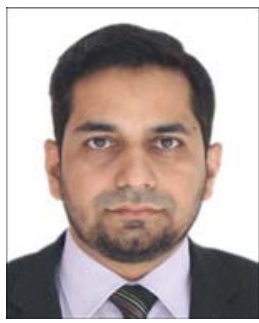

Faheem A. Khan (M'02) is currently a Senior Lecturer with the School of Computing and Engineering, University of Huddersfield, U.K. He received the $\mathrm{Ph} . \mathrm{D}$. degree in electrical and electronic engineering from Queen's University, Belfast, U.K., in 2012 In the past, he worked as a Postdoctroral Research Associate with the Institute for Digital Communications, University of Edinburgh, U.K., where he contributed to research in EU research projects HARP and ADEL. He has authored or co-authored more than 20 papers in refereed journals and conferences. His research interests include the field of wireless communications and signal processing with particular focus on cognitive radio, MIMO, and millimeterwave communications. He has significant previous teaching and research experience at academic institutions in the U.K., Middle East, and India. He is a Fellow of the Higher Education Academy, U.K. 